\title{
Heading backward: Perceived direction of movement in contracting and expanding optical flow fields
}

\author{
DIRK KERZEL \\ University of Bielefeld, Bielefeld, Germany \\ and \\ HEIKO HECHT \\ Center for Interdisciplinary Research \\ University of Bielefeld, Bielefeld, Germany
}

\begin{abstract}
Two goals were pursued in an investigation of possible visual sources for directionality judgments of ego-motion. First, J. J. Gibson's (1950) global radial outflow hypothesis was contrasted with a simple extrapolation strategy. Second, backing-direction judgments capitalizing on the informational equivalence of global radial outflow created during forward ego-motion and global radial inflow during backward ego-motion were explored. In comparing the accuracy of heading and backing judgments, new insights about global flow and extrapolation strategies were found. Consistent with the hypothesis of an extrapolation strategy, Experiment 1 demonstrated that backing judgments were more accurate than heading judgments when linear observer motion was simulated by means of a point-light flow field. In this case, accuracy was higher with two-point-light displays (extrapolation) than with more complex displays (global flow). Experiment 2 showed that in cases where extrapolation was not possible, such as circular motion, no advantage of backing judgments could be found and judgments were generally less accurate. We conclude that, whenever possible, observers use extrapolation to determine their heading/backing. Only when global flow is the only good source of information do they rely on it.
\end{abstract}

The ego-motion of an observer through a rigid environment creates an optical flow field with the following characteristics: The rate of optical flow is minimal at a point directly in front of the observer, typically corresponding to her/his direction of heading during forward movement. The optical field expands away from a focus of expansion (FOE). The rate of optical flow is also minimal directly behind the observer, where the field contracts toward the focus of contraction (FOC). The FOC is visible to an observer only if she/he is moving backward. In between these nodal points, the rate of optical flow is maximal directly underneath the observer (for detailed discussions, see J. J. Gibson, 1950; Koenderink, 1990; R. Warren, 1990; W. H. Warren, 1995).

It has been suggested that human locomotion and orientation make use of these facts. In particular, the re-

This work was supported by an equipment grant (He 2122/4-1 367/95) from the Deutsche Forschungsgemeinschaft to the second author and a fellowship (Studienstiftung des Deutschen Volkes) to the first author. We thank Mary Kaiser and Nam-Gyoon Kim for valuable comments. We also thank Nicola Bruno, James Cutting, and one anonymous reviewer for their comments on an earlier version of this paper. D. Kerzel is now at the Max-Planck Institute for Psychological Research in Munich. Correspondence should be addressed to H. Hecht, ZiF/Universität Bielefeld, Wellenberg 1, 33615 Bielefeld, Germany (email: hecht@hrz.uni-bielefeld.de). markably good ability to judge heading direction has been explained as the extraction of global information from optical flow (W. H. Warren, Morris, \& Kalish, 1988). However, there is recent evidence that local cues can be very important and can influence heading judgments even in the presence of simultaneous global flow-field information. For example, Cutting (1996) found that local displacement cues were exploited to a higher degree than were global information sources when observers had to judge heading direction in a cluttered environment. Thus far, theories of wayfinding have adopted a paradigm of heading-direction judgments. In contrast, we investigated the ability of observers to extract backing information, which is optically equally as well specified as is heading information. An anisotropy between heading and backing judgments was found.

\section{Theoretical Equivalence of Heading and Backing}

Assuming pure observer translation through a rigid environment, J. J. Gibson (1950) identified the focus of radial outflow, or FOE, as the point in the field of optical flow to which the observer is moving. Similarly, the direction from where the observer is coming is specified by the focus of radial inflow, or FOC. J. J. Gibson emphasized that the direction of heading (or backing if the observer moves backward) could be derived from any point in the flow field. It is thus the global flow pattern 
that specifies heading. If observers do use the global flow field to determine heading/backing, it should be more difficult to extract the FOE when the number of visible elements is reduced but easier when the number of elements is increased. This was, in fact, found to be the case by W. H. Warren et al. (1988), and the finding was replicated by Cutting, Springer, Braren, and Johnson (1992).

However, although FOC and FOE are informationally equivalent, the study of backing has been neglected. To our knowledge, no systematic studies have been published that assess observers' abilities to judge the direction of self-motion (backing direction) on the basis of the optical flow that results from backward movement. The FOC may have been neglected because of the larger ecological significance of forward observer motion. Only in rare instances, as when looking out the rear window of a driving car, are we confronted with optical contraction. It is ironic that James Gibson, his father being a railroad man, might have been inspired to his analysis of optical flow by his experiences as a boy when standing on the rear platform of a moving train and looking backward (Boring \& Lindzey, 1967).

\section{Attunement Versus Extrapolation}

Given the ubiquitous nature of self-motion, our visual system should be well attuned to the optical flow patterns usually associated with observer movement (E. J. Gibson, 1969). In other words, our exposure to heading and backing information should have been sufficient to develop the faculty to extract the necessary optical invariants in both situations. Granting the comparatively infrequent experience of contracting optical flow, a direct perceptionist should predict that our ability to detect heading is better or at least as good as the ability to detect backing. And, in fact, in a different context, evidence has been found that performance is worse when the objects moved away from the eye than when they moved toward the eye (Perrone, 1986). The rigid 3-D structure of projected 2D wire-frame boxes was perceived veridically when the objects moved toward the observer, but distortions compatible with a 2-D interpretation of the objects were perceived when they moved away from the observer. It is not evident, however, if these results generalize to the more basic task of extracting heading/backing direction from an optical flow field.

A broad class of models of human heading perception emphasizes the informational importance of the global flow field (for an overview, see W. H. Warren, 1995). Differential models that determine heading from spatial derivatives of the flow field (Koenderink \& van Doorn, 1976, 1981; Longuet-Higgins \& Prazdny, 1980; Waxman \& Ullman, 1985) require a sufficiently continuous field and fail with sparse flow fields. A major problem of such differential models, their sensitivity to noise, was solved by using template models (see, e.g., Hatsopoulos \& W. H. Warren, 1991; Perrone \& Stone, 1994). These models also have the advantage that they do not require a large number of points. A few points suffice to guide the search for motion parameters that optimally explain the flow pattern. A somewhat different approach was taken by Cutting's (1986) nondecompositional differential motion parallax model. It proceeds by evaluating a sufficient number of elements along the line of sight in order to identify the fastest one. Direction of heading is given by the direction opposite to the movement of the fastest element.

In computational vision, yet another route to the detection of heading has been taken. Algorithms capitalizing on motion parallax find the FOE/FOC by intersecting a set of lines determined by a number of flow vectors. Older versions of these algorithms assume a lower limit of depth variation for nearby points (Rieger \& Lawton, 1985), whereas more recent versions work without this restriction (Heeger \& Jepson, 1992). The flow lines trace the trajectory of single elements in the scene, and their extensions meet at the FOE/FOC. In a typical computergenerated heading experiment, one can obtain these lines by not deleting the video buffer such that consecutive pictures appear simultaneously on the screen.

An even simpler approach to the problem can be sought in local extrapolation processes, which might be more akin to human vision. The above algorithms can be interpreted as corresponding to a strategy whereby observers extract translational heading by comparing the optical velocities of two elements. The intersection of their flow vectors is extrapolated. A schematic diagram of such an extrapolation strategy is shown in Figure 1. Extrapolation has the advantage that it can be carried out in a flow field containing as few as two dots. 'The direction of heading/ backing is found by extrapolating the visible portion of the respective trajectories and finding their intersection.

Within such an extrapolation paradigm, a clear anisotropy between heading and backing is predicted. In the

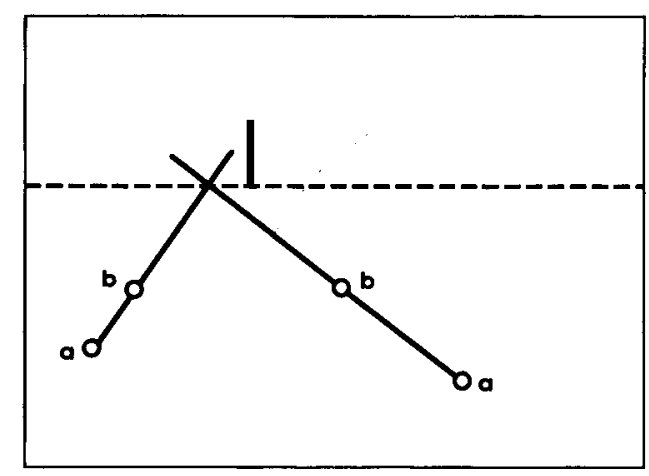

Figure 1. Experiment 1: Schematic drawing of extrapolated point-light trajectories (for two-dot cases only) that specify a heading/backing direction toward the left of the reference mark. For backing judgments, the point-lights moved from position a to position b, making extrapolation easy. For heading judgments, they moved from position $b$ to position a. The horizon is indicated by the dotted line in the drawing, but was not visible during the experiment. 
case of heading, the extrapolation task has to be done in a direction opposite to the motion of the elements, because the picture elements move away from the FOE. However, usually, human observers are engaged in extrapolating in the direction of motion, as, for example, when predicting the time to contact (Lee, 1976) or the trajectory of an object dropped from a moving carrier (McCloskey, Washburn, \& Felch, 1983). In the case of a person moving backward, picture elements of the resulting flow field move toward the FOC. Here, extrapolation in the direction of element motion would be required, making it easier to identify the FOC. In other words, local extrapolation strategies become easier within a backing paradigm. The models of heading perception presented above are indifferent as to the direction of motion.

Moreover, given a standard task in which observers watch a flow pattern of point lights for a short period of time and then indicate their direction of heading, the perceptual support is better for backing than it is for heading situations. The observer's gaze is naturally directed toward the FOC when pursuing the relevant target dots within the backing context. Conversely, in a heading context, there is no natural direction toward the FOE. Also, as Köhler (1931) has shown, length comparisons between line segments are less accurate, the larger the retinal distances subtended between them. Thus, for a given point-light, as its distance to the FOE is continuously increasing, it becomes progressively difficult to find the intersection of the flow vectors. The opposite is true for a point-light and its relation to the FOC. One could also entertain the cognitive notion that subjects can capitalize on representational momentum (Freyd \& Johnson, 1987) in the direction of element motion to determine the FOC but not the FOE.

In sum, the attunement to global flow-field structure predicts equal or worse performance in detecting the direction of backing than in detecting the direction of heading. The use of an extrapolation strategy, on the other hand, predicts better performance in backing than in heading tasks.

W. H. Warren et al. (1988) have hypothesized that if the visual system performs a global extraction of heading, judgments should be more accurate in dense flow fields. If, on the other hand, observers use an extrapolation strategy, judgment accuracy should increase, the fewer the number of point-lights making up the flow field. W. H. Warren et al. examined heading judgments during simulated observer translation parallel to a randomdot plane, varying observer speed and dot density. Using a forced-choice discrimination task, they found that performance dropped significantly with only two pointlights. They concluded that the determination of heading was based on the global radial outflow, as proposed by J. J. Gibson (1950), and not on extrapolation. This conclusion, however, might have been unwarranted, if our presumptions are true that extrapolation is particularly hard within a heading paradigm. W. H. Warren et al. may have made an unbalanced comparison. Adding a backing condition to their point-light numerosity manipulation cures this imbalance.

If observers do, in fact, extract global flow information when making heading/backing judgments, performance should be superior when the observers are not forced to use an extrapolation strategy. That is, both FOC and FOE should be best extracted with displays with many point-lights. If, on the other hand, observers use an extrapolation strategy, direction of backing should be detected more efficiently than direction of heading with displays with few point-lights.

\section{EXPERIMENT 1 Linear Paths}

In Experiment 1, we sought to evaluate the role of extrapolation strategies in the detection of heading/backing. To this end, W. H. Warren et al.'s (1988) first experiment was replicated as closely as possible. In addition, the direction of observer motion (heading vs. backing) was varied. The four independent variables that were manipulated by W. H. Warren et al. and in the present experiment were density (only the highest and lowest densities were included), target angle, and heading angle. For half of the observers, speed was kept constant at a somewhat higher speed than it was by W. H. Warren et al., while, for the other half, speed was identical to the fastest speed condition used by W. H. Warren et al. With slower velocities, shorter portions of the dots' paths are shown and their screen velocities are lower. Thus, less information about the motion direction of each individual dot is given. This should hypothetically lead to a decline in performance in the two-dot condition in both heading and backing trials.

\section{Method}

Subjects. Twenty students at the University of Bielefeld ( 15 males and 5 females) were paid for their participation. All had normal or correctedto-normal vision and all were naive as to the purpose of the experiment.

Stimuli and Apparatus. The experiment was run on a Silicon Graphics Indigo $2 \mathrm{XZ}$ workstation. The stimuli were displayed on a $48-\mathrm{cm}$ (diagonal) color monitor with a resolution of $1,280 \times 1,024$ pixels and a refresh rate of $72 \mathrm{~Hz}$. The update rate of the animation was $72 \mathrm{frames} / \mathrm{sec}$.

All displays consisted of a plane of point-lights whose projected size remained constant. They simulated a translation of the observer parallel to a horizontal random dot plane. Although the geometry of the displays was ambiguous with regard to absolute sizes and distances, we presented computer simulations consistent with one possible environment. The flow field subtended a visual angle of $17.4^{\circ}$

Design. A $2 \times 2 \times 2 \times 4 \times 10$ mixed factorial design was used. One between-subjects factor and four within-subjects factors were manipulated. The first factor, simulated observer's speed, was varied between subjects. An equal number of subjects saw displays simulating motions of 3.8 and $7.6 \mathrm{~m} / \mathrm{sec}$. During the $2.6 \mathrm{sec}$ of each animated sequence ( 187 frames), observers were simulated to be moving over distances of 9.85 and $19.7 \mathrm{~m} / \mathrm{sec}$, respectively. The second factor was the direction of the simulated observer's motion. Either a forward motion or a backward motion was simulated. Conditions on the factor were blocked and the order of presentation was counterbalanced across subjects. 
The third factor was the density of dots. In the many-dot condition, the density was .12 dots $/ \mathrm{m}^{2}$ in virtual space. The dots were placed randomly on the simulated ground surface with the constraint that only one dot be placed in each cell of a virtual grid on this surface. To avoid a dense clustering of dots on the horizon, the ground plane was clipped at a simulated distance of $38 \mathrm{~m}$. Thus, the horizon line was not visible. However, the size of the simulated ground plane exceeded the clipping distance such that the visual angle subtended by the flow field remained at its initial size during the simulated observer's motion. In the two-dot condition, one dot was placed on either side of the heading vector. The distance of the two dots from the observer and the offset from the heading vector were constrained such that the dots remained visible on the screen throughout the trial. This had the effect that the initial dot positions in heading trials, as well as the final dot positions in backing trials, subtended a visual angle not exceeding $5.8^{\circ}$ at a speed of $3.8 \mathrm{~m} / \mathrm{sec}$ and $3.7^{\circ}$ at a speed of $7.6 \mathrm{~m} / \mathrm{sec}$. The fourth factor was the position of a vertical target line that appeared at the end of each trial. The target could appear either $\pm 6^{\circ}$ or $\pm 2^{\circ}$ from the center of the screen. The last factor was the heading angle with respect to the target. This was determined by the direction of translation, and it varied between $\pm 0.2^{\circ}$, $\pm 0.5^{\circ}, \pm 1.0^{\circ}, \pm 2.0^{\circ}$, and $\pm 4.0^{\circ}$

Thus, subjects saw a total of 160 trials consisting of the factorial combination of 2 heading directions (forward, backward) $\times 2$ densities (many dots, two dots) $\times 4$ target positions $\left( \pm 6^{\circ}, \pm 2^{\circ}\right) \times 10$ heading angles $\left( \pm 0.2^{\circ}, \pm 0.5^{\circ}, \pm 1.0^{\circ}, \pm 2.0^{\circ}\right.$, and $\left.\pm 4.0^{\circ}\right)$.

Procedure. The experiment took place in a dimly lit room. The subjects viewed the screen monocularly with the right eye from a distance of $40 \mathrm{~cm}$. Head movements were restricted by a chinrest. The subjects initiated each trial by pressing the space bar. On each trial, after the first frame of each trial remained visible for $1 \mathrm{sec}$ as a warning signal, the animation started and moved for $2.6 \mathrm{sec}$. The last frame remained visible until a binary response was made, which indicated the perceived direction of heading. By pressing one key, the subject indicated that she/he was going to or coming from the left of the target and, by pressing another key, indicated a perceived FOC or FOE to the right of the target line. The trials were presented in different random orders for each subject. The data were collapsed across positive/negative target positions and positive/negative heading angles. The subjects saw a set of 12 practice trials. The experimental session took about $30 \mathrm{~min}$.

\section{Results and Discussion}

Overall accuracy was in the same range as that reported by W. H. Warren et al. (1988). Consistent with the extrapolation hypothesis, backing judgments were more accurate than heading judgments and judgments with just two dots were more accurate than those with many dots. Similar to W. H. Warren et al. (1988), we found accuracy to be higher with fast screen velocities.

An analysis of variance (ANOVA) was performed on the full factorial design. As indicated in Figure 2, subjects' performance in the condition with fast screen velocities was better than that with slow velocities $[F(1,18)=4.8$, $p=.0438]$.

Figures $2 \mathrm{~A}$ and $2 \mathrm{~B}$ show that the detection of heading/ backing was easier in the two-dot condition $[F(1,18)=$ $31.08, p<.0001]$ than in the many-dot displays. Mean judgment accuracy is plotted for each density and speed condition. The mean $75 \%$-correct threshold was at $.65^{\circ}$ in the two-dot condition and at $1.3^{\circ}$ in the many-dot condition.

Backing judgments were more accurate than heading judgments $[F(1,18)=6.7, p=.0186]$. Mean percent correct judgments are presented as a function of heading angle for each motion direction and speed condition in Figures $2 \mathrm{C}$ and $2 \mathrm{D}$. The mean $75 \%$-correct threshold was at $1.2^{\circ}$ in the heading condition and at $0.75^{\circ}$ in the backing condition. A main effect of heading angle was

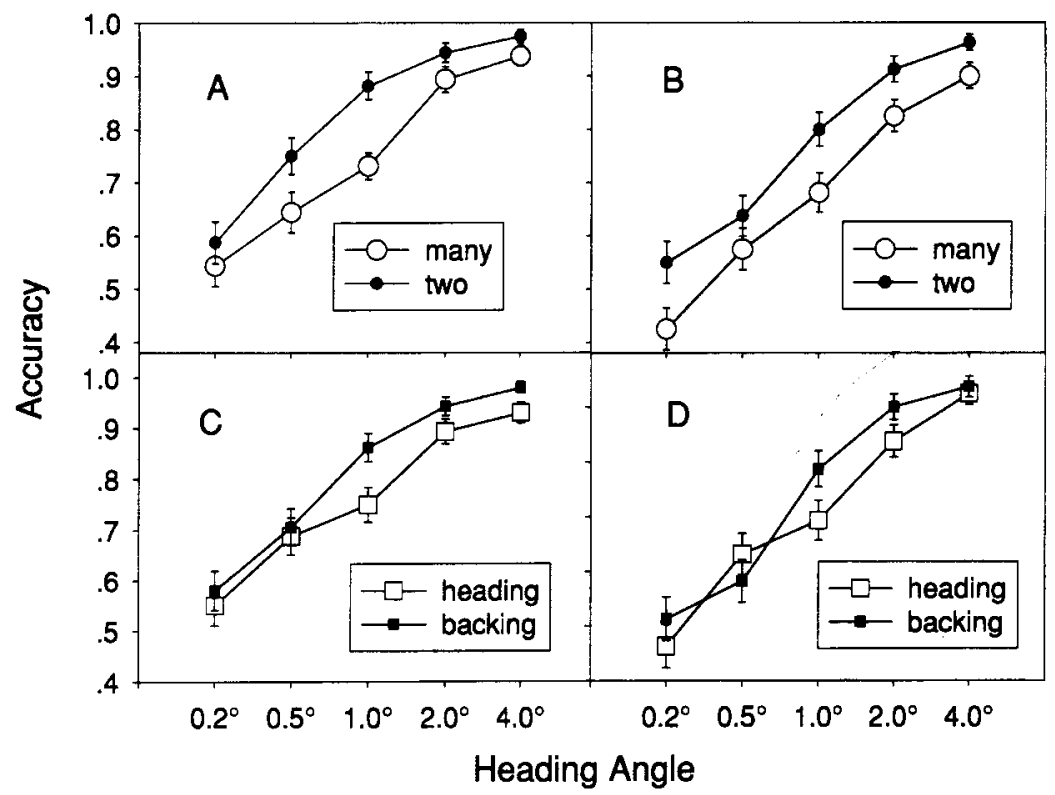

Figure 2. Experiment 1 : Average judgment accuracy as a function of heading angle and dot density is shown for high simulated velocities (panel $A$ ) and slow velocities (panel B). Average judgment accuracy as a function of heading angle and direction of motion is shown for high simulated velocities (panel C) and slow velocities (panel D) (correct heading judgment $=1$, incorrect heading judgment $=0$ ). 
found; judgments were more accurate the farther the reference mark was from the FOC or FOE $[F(4,72)=$ $141.14, p<.0001]$. No significant interactions were found.

Additionally, two separate three-way repeated measures ANOVAs were performed on the data from the two speed conditions. The superiority of backing trials was reliable for fast screen velocities $[F(1,9)=8.74, p=$ $.016]$ but not for slow velocities $[F(1,9)=1.43, p=$ .262]. Detection of heading/backing was easier in the two-dot condition for both fast velocities $[F(1,9)=$ $14.09, p=.0045]$ and slow velocities $[F(1,9)=17, p=$ $.0026]$. A main effect of heading angle was found in both speed conditions [with fast velocities, $F(4,36)=76.74$, $p<.0001$; with slow velocities, $F(1,9)=67.06, p<$ $.0001]$. No significant interactions were found.

Some subjects reported using a strategy of finding the intersection of two lines drawn by the dots. This strategy was easy to apply in the two-dot condition, whereas, in the many-dot condition, the flow field was likely to be distracting. However, all reported a stronger sense of ego-motion in the many-dot conditions.

In contrast to W. H. Warren et al. (1988), judgments were more accurate in the two-dot condition. The superiority of global flow was not replicated; we obtained the opposite effect. This result could be explained by the use of an extrapolation strategy but not by global flow analysis. The conditions for extrapolation differ in two important ways from global-flow analysis. First, in the absence of a visible horizon, two dots are necessary and sufficient for extrapolation. Second, dots used for extrapolation have to be singled out. In the many-dot condition, if the observer determines heading by triangulating, two or more dots have to be chosen from a large set of visible dots. However, most of these dots are likely to go past the near or far clipping distance during the animated sequence. Unless dots that remain on the screen during the complete sequence are accidentally focused, refocusing is necessary. This is likely to interfere with performance as compared with that in the condition in which two dots are presented and remain on screen during the complete sequence. The relatively large dot-density effect with faster screen velocities can be explained by the fact that more dots have to be replaced, thus requiring more refocusing. Note that in both W. H. Warren et al.'s and the present experiment, only the two-dot trials were constrained such that both dots remained visible during the whole trial.

These results are at odds with J. J. Gibson's (1950) global FOE/FOC hypothesis, which makes the opposite prediction for the two density conditions. If the extraction of heading was dependent on the global flow field, then performance should deteriorate in the two-dot condition. The fact that this pattern of results was found by W. H. Warren et al. (1988) and by Cutting et al. (1992) but not in the present experiment can be attributed only to the lower simulated speeds in W. H. Warren et al.'s first ex- periment. The only marked difference between our heading condition and those two studies were the comparatively fast screen velocities in our displays and the smaller number of trials containing global flow-field information. In the fast-speed condition, screen velocities were twice W. H. Warren et al.'s speeds and about three times as fast as the simulations used by Cutting et al. At higher speeds, the displacement of the projected dots per unit of time is greater-that is, the magnitude of the velocity vectors on the projection screen is higher. Therefore, a longer sequence of the dots' path is shown to the observer, which facilitates identification of the velocity vectors and, therefore, extrapolation.

Other traditional hypotheses in this domain also fail to explain the effects: Cutting's (1986) differential motion-parallax hypothesis, Koenderink and van Doorn's (1981) local maximum of divergence hypothesis, and template models cannot account for the observed anisotropy between heading and backing. They are also clearly at odds with the superiority of the two-dot condition.

One might argue that the constrained motion (within $3.7^{\circ}$ and $5.8^{\circ}$ of visual angle) of two-dot trials was responsible for their superiority over many-dot trials. However, this should have, above all, facilitated large heading angles, which it did not do. The difference between two-dot and many-dot trials was significant for heading angles smaller than $4^{\circ}$ (see Figure 2 ).

As the separate ANOVAs show, there was no effect of direction of motion with slow screen velocities. Thus, the effect might be obtainable only at high screen velocities when extrapolation is particularly facilitated by virtue of fast tracking movements, which point toward the FOC and not away from it as in the heading case.

In sum, the findings are consistent with the hypothesis that observers make use of an extrapolation strategy and are clearly at odds with J. J. Gibson's (1950) hypothesis that global flow invariants are used for heading/ backing judgments.

\section{EXPERIMENT 2 Circular. Paths}

It was found in Experiment 1 that, for fast screen velocities, the identification of backing is easier than the identification of heading. This result has been interpreted as evidence for an extrapolation strategy. However, it might be that ego-motion is easier to detect during backward motion at high velocities. To rule out this explanation, we decoupled heading/backing and FOE/ FOC by comparing the accuracy of heading and backing judgments during observer motion with and without a visible FOC or FOE. To this end, W. H. Warren, Mestre, Blackwell, and Morris's (1991) first experiment was replicated, simulating movements on circular trajectories.

A circular movement of the observer may be analyzed into a translational and a rotational component. To re- 
cover heading, extrapolation is not a feasible strategy, because the lines specified by the translational components of the velocity vectors do not intersect at a single point. Nonetheless, observers have been shown to perceive their heading accurately on circular trajectories (W. H. Warren et al., 1991; Stone \& Perrone, 1997). Projectively, a circular motion gives rise to a steady flow, a stationary field with constant streamlines. The streamline that passes beneath the observer specifies the observer's future path on the ground plane. Lee and Lishman (1977) called this streamline "locomotor flow line." However, W. H. Warren et al. have shown that heading accuracy remains high with as few as two dots and conclude that circular heading is specified by the motions of a few elements. Consequently, locally defined features of the flow pattern such as the locomotor flow line are unnecessary.

\section{Method}

Stimuli and Apparatus. The apparatus was the same as that used in Experiment 1. Displays simulated a circular movement parallel to the random-dot ground plane. The density of the simulated surface was $0.12 \mathrm{dots} / \mathrm{m}^{2}$. That is, only many-dot trials were presented. The tangential velocity was fixed at $7.6 \mathrm{~m} / \mathrm{sec}$

Design. A $2 \times 8 \times 8$ factorial design was used. The first factor was the direction of the simulated observer motion. Either a forward motion or a backward motion was simulated. Conditions were blocked, and the order of presentation was balanced across subjects. The second factor was the radius of the observer's path, which varied randomly among $\pm 40, \pm 80, \pm 160$, and $\pm 320 \mathrm{~m}$. Positive values indicate a right turn, and negative values a left turn. The virtual observer was always rotated about an axis that was perpendicular to the ground plane. The third factor was the heading angle - that is, the visual angle between a target on the ground surface and the point at which the observer's path would pass the target, determined from the simulated observation point at the end of the display. The latter point was defined as the point on the path from which a line to the observation point was perpendicular to a line to the target. The target was a vertical line segment that appeared in the last frame of the display at a distance of approximately $16 \mathrm{~m}$ from the observer. Heading angle varied randomly at $\pm 0.5^{\circ}, \pm 1.0^{\circ}, \pm 2.0^{\circ}$, and $\pm 4.0^{\circ}$ such that the path of the observer was directed to either the inside or the outside of the target.

Thus, subjects saw a total of 128 trials consisting of the factorial combination of 2 heading directions (forward, backward) $\times 8$ radii $( \pm 40, \pm 80, \pm 160$, and $\pm 320 \mathrm{~m}) \times 8$ heading angles $\left( \pm 0.5^{\circ}, \pm 1.0^{\circ}\right.$, $\pm 2.0^{\circ}$, and $\pm 4.0^{\circ}$.

Procedure. The procedure was the same as that used in Experiment 1. The data were collapsed across left and right turns and positive and negative heading angles. Every observer saw a set of 16 practice trials, followed by a different random order of all experimental trials. The experimental session lasted for about $30 \mathrm{~min}$.

\section{Results and Discussion}

The overall accuracy was comparable to that of W. $\mathrm{H}$. Warren et al. (1991). Consistent with the notion that the identification of heading is not generally easier during backward motion, no difference emerged between heading and backing judgments.

A repeated measures ANOVA, including all factors, was performed. A main effect of radius $[F(3,27)=7.29$ $p=.001]$ revealed that small radii (i.e., strong curvature) made heading/backing judgments more difficult. Again, an effect of heading angle $[F(3,27)=31.95, p<.0001]$

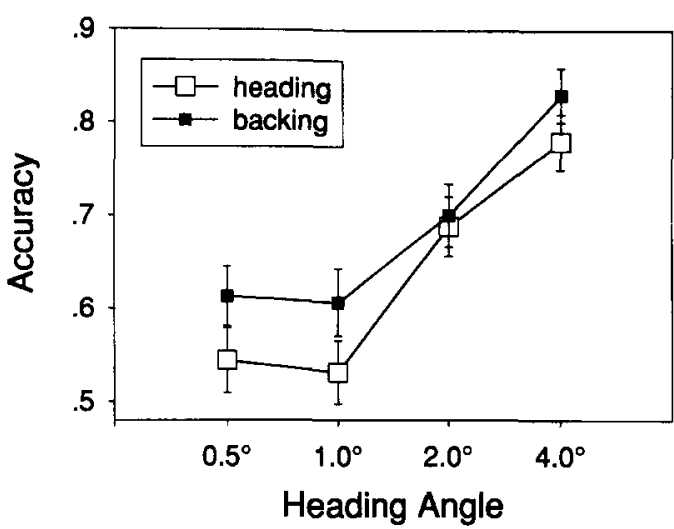

Figure 3. Experiment 2: Average judgment accuracy as a function of heading angle and direction of motion. Error bars indicate standard errors of the mean.

was found; accuracy increased with distance to the reference mark. The mean percent-correct judgments are presented as a function of heading angle and direction of motion for each radius condition in Figure 3. The mean $75 \%$-correct threshold was $3^{\circ}$. The effect of direction of motion was not significant $[F(1,9)=2.29, p=.164]$; that is, no difference between heading and backing judgments was found. For radii of 40 and $80 \mathrm{~m}$, performance was better when the target was placed on the inside of the trajectory $[t(318)=-7.04, p<.0001$, and $t(318)=-3.4, p=$ .0007 , respectively], indicating a bias to perceive the direction of heading toward the outside of the actual trajectory. For radii of $40 \mathrm{~m}$ (and $80 \mathrm{~m}$ ), mean percent correct were $40 \%(54 \%)$ when the target was placed on the outside and $76 \%(72 \%)$ when it was placed on the inside.

Although a stable FOE/FOC was lost, heading and backing judgments were quite accurate. Since the circular motion trajectories prevented the use of an extrapolation strategy, the heading/backing information that observers extracted from these displays must have been based on global optical flow. Thus, in the absence of local information, observers made use of global flow. This would also explain why the differential effects of heading and backing disappeared. Compatible with the results found by W. H. Warren et al. (1991), accuracy decreased with smaller radii. The absence of an effect of the direction of motion supports the hypothesis that it is not generally easier to detect the direction of locomotion when going backward.

\section{GENERAL DISCUSSION}

The informational analysis of optical flow due to translational egomotion is symmetric in the sense that the direction of backing (of a backward-moving observer) is equally well specified as the direction of heading (of a forward-moving observer). In conflict with this informational symmetry, empirical judgments of heading and backing in simulated flow fields showed a clear anisotropy. Surprisingly, heading judgments were less accurate than backing judgments in corresponding flow fields simulating a high-velocity observer motion. This anisotropy 
suggests that observers do not always base their judgments on global optical flow as originally hypothesized by J. J. Gibson (1950).

Instead, the displays that we used to simulate linear observer trajectories produced results consistent, for two reasons, with the use of an extrapolation strategy. First, such a strategy favors backing judgments because extrapolation is facilitated by shrinking distance between the elements and the FOC, by a more natural direction of extrapolation, or even cognitive processes of representational momentum. However, this effect was obtainable only at high screen velocities. Perhaps tracking made the FOC (and not the FOE) particularly salient and thus contributed to the advantage of heading over backing. At slower speeds, this advantage is lost because tracking movements become slower. Second, regardless of speed, judgments were more accurate in the two-dot condition than in the many-dot condition. This finding is at odds with the hypothesis that observers rely exclusively on the global flow pattern when judging heading. It supports the idea that two dots are sufficient for extrapolation, while more dots might lead to distraction.

The conclusion is qualified by the significant effect of simulated observer speed. In the context of a slow flow field, observers do not seem to apply extrapolation strategies. Their performance in these cases is best explained by a global flow analysis. However, even at moderate screen velocities, our results contradict the findings obtained by W. H. Warren et al. (1988). One explanation of W. H. Warren et al.'s failure to find a superiority of the two-dot condition might lie in the strategy that was imposed by the study. Only half of our trials had global flow information, whereas $75 \%$ of W. H. Warren et al.'s trials contained such global information. Thus, a global strategy might have been profitable only in the context of their study. This does, of course, presuppose that observers can and do switch between a global flow-field analysis and a local extrapolation strategy.

In agreement with W. $\mathrm{H}$. Warren et al. and colleagues (W. H. Warren, 1988; W. H. Warren et al,, 1991), our results indicate that the extraction of a focus of expansion or contraction plays an important role for heading and backing judgments. In Experiment 2, we replicated the result that curved trajectories produce less accurate heading judgments (compare Figures $2 \mathrm{C}$ and 3 ) than do linear trajectories. Moreover, the results demonstrate that the identification of backing is not generally more accurate than the identification of heading, as Experiment 1 might have suggested. Thus, the anisotropy between heading and backing was limited to linear observer trajectories and consequently with stationary FOE and FOC.

In our displays (Experiment 1), observers were able to use extrapolation with good results, and they seem to have been confused the more point-lights they could choose from to do the task. In W. H. Warren et al.'s (1988) displays, on the other hand, screen velocities were comparatively slow, presumably so slow that extrapolation could not be used efficiently. In that case, however, observers were able to make use of the global flow-field information, which resulted in better performance when the stimulus consisted of many point-lights. An additional indication that this might be the case is W. H. Warren et al.'s (1988, first ex periment) finding of an interaction between the speed of the animation and the density of the flow field. Their observers were presumably more accurate when few point-lights were shown at high speeds.

If this interpretation is true, the following conclusions can be drawn The question of whether observers extract information from global flow or use local cues is mediated by the speed of optical flow. When observers are prevented from using extrapolation-as in the case of slow screen velocities or of curved trajectories - they are able to base their judgments on global optical flow, albeit with considerably less accuracy. The lack of a difference between backing and heading judgments, as found in the slow-speed condition of Experiment 1, lends support to this interpretation. If, on the other hand, motion extrapolation is possible - with fast screen velocities and straight trajectories - observers use an extrapolation strategy, as supported by the fact that backing judgments are more reliable than heading judgments at high speeds. In this case, the use of extrapolation can be hampered by clutter in the display, and many point-lights do not help but seem only to confuse. Observers may also fail to extrapolate locally if they are in the mode of expecting information that is globally specified.
For curved trajectories, no difference between the two-dot and the many-dot conditions was found by W. H. Warren et al. (1991). This supports the notion that even in the many-dot case observers may not extract information from global flow but use some other image-based strategy. Interestingly, W. H. Warren et al. (1991) neither interpreted this lacking effect of dot density nor entertained the possibility of the existence of other strategies that were not based on global flow.

In conclusion, observers' backing judgments are as accurate as heading judgments and, in the case of fast simulated velocities, backing is even superior to heading regardless of the dot density of the display. Observers are able to base heading and backing judgments on local information (two-dot displays) and often adopt an extrapolation strategy. However, this does not preclude the use of global flow-field information in those cases where extrapolation is difficult or in richer naturalistic settings.

\section{REFERENCES}

BORING, E. G., \& LINDZEY, G. (Eds.) (1967). A history of psychology in autobiography (Vol. 5, pp. 125-143). New York: Appleton-CenturyCrofts.

Cutting, J. E. (1986). Perception with an eye for motion. Cambridge, MA: MIT Press.

Cutting, J. E. (1996). Wayfinding from multiple sources of local information in retinal flow. Journal of Experimental Psychology: Human Perception \& Performance, 22, 1299-1313.

Cutting, J. E., Springer, K., Braren, P. A. \& Johnson, S. H. (1992). Wayfinding on foot from information in retinal, not optical, flow. Journal of Experimental Psychology: General, 121, 41-72.

Freyd, J. J., \& Johnson, J. Q. (1987). Probing the time course of representational momentum. Journal of Experimental Psychologv: Learning, Memory, \& Cognition, 13, 259-268.

Gibson, E. J. (1969). Principles of perceptual learning and development. Englewood Cliffs, NJ: Prentice-Hall.

GiBson, J. J. (1950). The perception of the visual world. Boston: Houghton Mifflin.

Hatsopoulos, N. G., \& WARREN, W. H.(1991). Visual navigation with a neural network. Neural Networks, 4, 303-317.

HeEger, D. J., \& JePSON, A. D. (1992). Subspace methods for recovering rigid motion: I. Algorithm and implementation. International Journal of Computer Vision, 7, 95-117.

KoENDERINK, J. J. (1990). Some theoretical aspects of optical flow. In R. Warren \& A. H. Wertheim (Eds.), Perception and control of selfmotion (pp. 53-68). Hillsdale, NJ: Erlbaum.

KoEnderink, J. J., \& VAN Doorn, A. J. (1976). Local structure of movement parallax of the plane. Journal of the Optical Society of America, 66, 717-723.

KoEnderink, J. J., \& van DoOrn, A. J. (1981). Exterospecific component of the motion parallax field. Journal of the Optical Society of America, 71, 953-957.

KöHLER, W. (1931). Zur Psychophysik des Vergleichs und des Raumes. Psychologische Forschung, 14, 343-360.

LEE, D. N. (1976). A theory of visual control of braking based on information about time-to-collision. Perception, 5, 437-459.

Lee, D. N., \& Lishman, R. (1977). Visual control of locomotion. Scandinavian Journal of Psychology, 18, 224-230.

Longuet-Higgins, H. C., \& Prazdny, K. (1980). The interpretation of a moving retinal image. Proceedings of the Royal Society of London: Series $B, \mathbf{2 0 8}, 385-397$.

McCloskey, M., WashbuRn, A., \& FelCh, L. (1983). Intuitive physics: The straight-down belief and its origin. Journal of Experimental $P s y-$ chology: Learning, Memory, \& Cognition, 9, 636-649.

Perrone, A. J. (1986). Anisotropic responses to motion toward and away from the eye. Perception \& Psychophysics, 39, 1-8.

Perrone, A. J., \& Stone, L. S. (1994). A model of self-motion estimation within the primate extrastriate visual cortex. Vision Research, 34, 2917-2938.

Rieger, J. H., \& LaWTon, D. T. (1985). Processing differential image motion. Journal of the Optical Society of America A, 2, 354-360. 
Stone, L. S., \& Perrone, J. A. (1997). Human heading estimation during visually simulated curvilinear motion. Vision Research, 37, 573590.

WARREN, R. (1990). Preliminary questions for the study of egomotion. In R. Warren \& A. H. Wertheim (Eds.), Perception and control of self-motion (pp. 3-32). Hillsdale, $\mathrm{NJ}$ : Erlbaum.

WARREN, W. H. (1995). Self-motion: Visual perception and visual control. In W. Epstein \& S. Rogers (Eds.), Perception of space and motion (pp. 263-325). New York: Academic Press.

Warren, W. H., Mestre, D. R., BlackWell, A. W., \& Morris, M. W. (1991). Perception of circular heading from optical flow. Journal of Experimental Psychology: Human Perception \& Performance, 17, $28-43$.

Warren, W. H., Morris, M. W., \& Kalish, M. (1988). Perception of translational heading from optical flow. Journal of Experimental Psychology: Human Perception \& Performance, 14, 646-660.

Waxman, A. M., \& Ullman, S. (1985). Surface structure and 3D motion from image flow: A kinematic analysis. International Journal of Robotics Research, 4, 72-94.

\section{NOTE}

1. If a fixed reference is given, such as a horizon line, even one dot suffices. We avoided presenting a horizon line by clipping the simulated ground plane at a given distance from the observer (see Experiment 1).

(Manuscript received January 16, 1997; revision accepted for publication June 5, 1997.) 